\section{A Review of the Prevalence and Associations of Depression and Anxiety in Type 1 Diabetes Mellitus}

\author{
Subasinghe $S^{1 *}$, Bongetti $E^{3}$, O’Brien $C^{1,2}$, Silberberg $C^{1,2}$, \\ Ward G3,4, Maclsaac $R^{3}{ }^{3,4,6}$, Jenkins $A^{3,4,5}$, O'Neal $D^{3,4}$, Best \\ $\mathrm{JD}^{3,4}$, Loh $\mathrm{MM}^{4}$, Salzburg $\mathbf{M}^{1,2}$, Ski CF${ }^{7}$, Thompson $\mathrm{DR}^{7}$ and \\ Castle D $D^{1,2}$
}

${ }^{1}$ St Vincent's Mental Health Service, St Vincent's Hospital, Fitzroy, Victoria, Australia

${ }^{2}$ Department of Psychiatry, The University of Melbourne, Fitzroy, Victoria, Australia

${ }^{3}$ Department of Medicine, The University of Melbourne, Fitzroy, Victoria, Australia

${ }^{4}$ Department of Endocrinology and Diabetes, St Vincent's Hospital, Fitzroy, VIC, Australia

${ }^{5}$ NHMRC Clinical Trials Centre, University of Sydney, Camperdown, NSW, Australia

${ }^{6}$ St Vincent's Institute of Medical Research, Fitzroy, Victoria, Australia ${ }^{7}$ Centre for the Heart and Mind, Australian Catholic University, Melbourne, Australia

\begin{abstract}
Aim: To perform a review of studies to identify the prevalence of depression and anxiety in adults with Type 1 diabetes mellitus compared to adults without Type 1 diabetes mellitus.

Methods: A computer search was conducted using the PubMed, EBSCOhost, PsychINFO, Cochrane, CINAHL and MEDLINE from 2009 onwards. The essential information extracted from the identified publications were: participant demographics; measurement tools; prevalence of depression; diabetes-specific and non-diabetes specific risk and protective factors for depression or anxiety in people with Type 1 DM; outcomes for Type 1 DM management and complications; and relevant qualitative data.

Results: An extensive literature search identified twenty-one key articles which were reviewed to provide strong evidence further
\end{abstract}

*Corresponding author: Subasinghe SS, St Vincent's Mental Health Service, St Vincent's Hospital, Fitzroy, Victoria, Australia, Tel: +61 039288 2211; E-mail: Supundi.subasinghe@svha.org.au

Citation: Subasinghe SS, Bongetti E, O'Brien CL, Silberberg C, Ward G, et al. (2015) A Review of the Prevalence and Associations of Depression and Anxiety in Type 1 Diabetes Mellitus. J Diabetes Metab Disord 2: 007.

Received: August 13, 2015; Accepted: December 03, 2015; Published: December 18, 2015

Copyright: (C) 2015 Subasinghe SS, et al., This is an open-access article dis tributed under the terms of the Creative Commons Attribution License, which permits unrestricted use, distribution, and reproduction in any medium, provided the original author and source are credited. supporting the notion that there is increased prevalence of comorbid depression and anxiety in Type $1 \mathrm{DM}$. Based on the literature reviewed, approximately one in five people living with Type 1 DM suffer from comorbid clinical depression, and one in four experience anxiety. There was variation in the exact rates reported between articles, however, which may be reflective of differences in methods or samples.

Conclusion: The pertinence of the evidence analyzed in this review is underpinned by findings from large international studies that have shown depression results in worse health outcomes for individuals compared to any other chronic disease, including diabetes. Further, the combination of depression and diabetes leads to even poorer

\section{Introduction}

Depression, anxiety and Type 1 Diabetes Mellitus (Type 1 DM) are frequently comorbid and this can significantly affect quality of life, glycaemic control, and other acute and chronic diabetes complications [1]. Depressive disorders have been found in more than one third of people with diabetes, and their treatment would improve the quality of life and health of people with Type 1 DM [2,3]. Anxiety, which is often comorbid with depression, is also more common in people with Type 1 DM compared to those without diabetes [4]. Comorbid depression or anxiety can increase challenges of self-management and are barriers to reaching treatment goals [5]. Moreover, the combination of depression with another chronic condition results in the largest decrement in overall health of any comorbidity combination [6]. This means that for people with Type $1 \mathrm{DM}$, having comorbid depression could be the most significant barrier to reaching optimal self-care. The significance of these negative implications is the resultant substantial healthcare costs, which account for over $\$ 3$ billion dollars annually in Australia [7]. Despite the significant burden that these conditions pose, comorbid depression and anxiety in diabetes is under-diagnosed and under-treated in Australia [8]. As a result numerous studies have been conducted to explore factors that may influence the association between these conditions.

The psychological impact of living with a chronic condition as well as biological factors relating to neurotransmitter metabolism and changes to the hypothalamic-pituitary axis have been implicated in the pathophysiology of comorbid depression and diabetes $[9,10]$. Raised levels of inflammation from hyperactivity of the hypothalamic-pituitary axis and insulin deficiency may underpin a biological dimension to this comorbidity in Type $1 \mathrm{DM}$ [4]. Concomitant psycho-biological changes secondary to depressive disorders can decrease motivation and increase sedentary behaviour, which are known to contribute to sub-optimal self-management [11]. Living with the diagnosis of a chronic condition is also a risk factor for mental illness [12]. Type 1 $\mathrm{DM}$ is a demanding condition that calls for persistent motivation and commitment even in the midst of competing life demands [13].

One of the most common sources of concern for people is the bidirectional relationship that occurs between the symptoms of diabetes and psychological stress [8]. For example, when blood glucose fluctuates too high or too low it can cause alterations in mood and cognition [14]; similarly the opposite can occur, as blood glucose 
can change in response to psychological stress often resulting in adjustments to insulin dose [15]. Key is that patients experiencing diabetes-distress and depressive symptoms experience difficulties in maintaining an effective management plan which has been linked to i) sub-optimal glycaemic control [16] and ii) higher incidence of diabetes-related complications [17]. Therefore psychological support is an essential component in holistic, multi-disciplinary diabetes care and should be considered a routine part of management $[2,8,18]$.

The crux of this issue lies within the unique challenge of diagnosing depression and anxiety in patients with chronic conditions. Patients with chronic conditions are generally less likely to be appropriately diagnosed with depression by their family doctor or their specialist than patients who do not have a chronic condition [19]. This could be due to a tendency to normalize psychological distress in the context of chronic conditions like Type 1 DM [20], especially when treatments are often perceived by healthcare professionals as being understandably burdensome to patients $[8,11]$. Increasing awareness and understanding of diabetes-specific stressors and common experiences can help differentiate 'normal' diabetes-related distress from pathological co morbid depression [21]. Past literature reviews have not included qualitative studies, however this data may provide essential insights [21,22] and help tailor diabetes-specific psych education, which is a key element in the treatment of depression and anxiety [18].

The majority of reviews on depression and/or anxiety and diabetes have focused on Type 2 Diabetes Mellitus (Type 2 DM) [23]. Given the psychological, physical and socioeconomic impact of comorbid depression and diabetes, and considering the differences in the average age of onset, pathophysiology, and management between Type $1 \mathrm{DM}$ and Type $2 \mathrm{DM}$, the current review of the associated literature will focus on Type 1 DM. Furthermore, there have been few reviews that have considered the prevalence of anxiety in Type $1 \mathrm{DM}$, and as most studies have been conducted outside Australia it is also unclear to what extent evidence can be generalized to our local population.

\section{Aim}

The aim of this review was to identify the prevalence, correlates and complications of depression and anxiety in adults with Type $1 \mathrm{DM}$ compared to adults without Type $1 \mathrm{DM}$.

\section{Methods}

A systematic electronic search was conducted to identify all relevant studies examining depression among adults with Type 1 DM. Inclusion criteria were: (i) Adults aged 19 years and older; (ii) Studies that examined people with Type $1 \mathrm{DM}$; studies that included participants with Type $2 \mathrm{DM}$ will be included if they adequately differentiate between the two groups in both their methods and results; (iii) Studies that assessed the effect of an intervention such as cognitive behavioural therapy or anti-depressant drugs will be included only if the effect of reducing depression or anxiety on diabetes (e.g., reducing complications or improving metabolic control) is measured; (iv) Type $1 \mathrm{DM}$ was formally diagnosed in participants or clarified with reliable medical records and studies used standardized measure of depression with established reliability and validity; (v) comparison with an appropriate control group or population norms.

\section{Exclusion criteria}

(i) literature reviews, case studies and qualitative research as these did not allow a prevalence rate of depression or the relationship between depressive symptoms and metabolic control to be extracted; (ii) Studies that include any participants under the age of 19 years; (iii) studies that only compared participants with Type $1 \mathrm{DM}$ to those with Type 2 DM; (iv) studies where depression was defined simply as those who take antidepressants.

\section{Search strategy}

A literature search was conducted between September and October 2013 on PubMed and EBSCOhost. The aim was to encapsulate as many relevant studies as possible, therefore relatively broad search terms were used. The searches were performed by searching MeSH term 'type 1 diabetes', with 'depressive disorder', 'anxiety', 'adjustment disorders', and 'distress'. The search was limited to show results that only included publications for the past five years, humans, adults (aged over 19 years), and studies published in the English language.

A second literature search was conducted in February 2014 (Figure 1), and in this instance PsychINFO, Cochrane, Pubmed, CINAHL and MEDLINE were used to ensure all relevant articles were captured Search terms were selected depending on the database, e.g., some databases had search headings that enabled researchers to specify specific disorders in the search, whereas others only allowed for the use of broad search headings such as 'depression' or 'anxiety'. For Pubmed, Cochrane and MEDLINE, the MeSH terms 'depression, 'depressive disorder, major,' 'dysthymic disorder,' 'depressive disorder, 'treatment resistant,' 'anxiety disorders,' 'adjustment disorders', were individually searched with the MeSH term 'diabetes mellitus, type 1' or titles including 'type 1 AND diabetes.' Separate searches for titles 'type 2 AND diabetes', and 'child* or adolescence*' were conducted and their results excluded from the main search results. A limit for articles published from 2009 onwards was then applied.

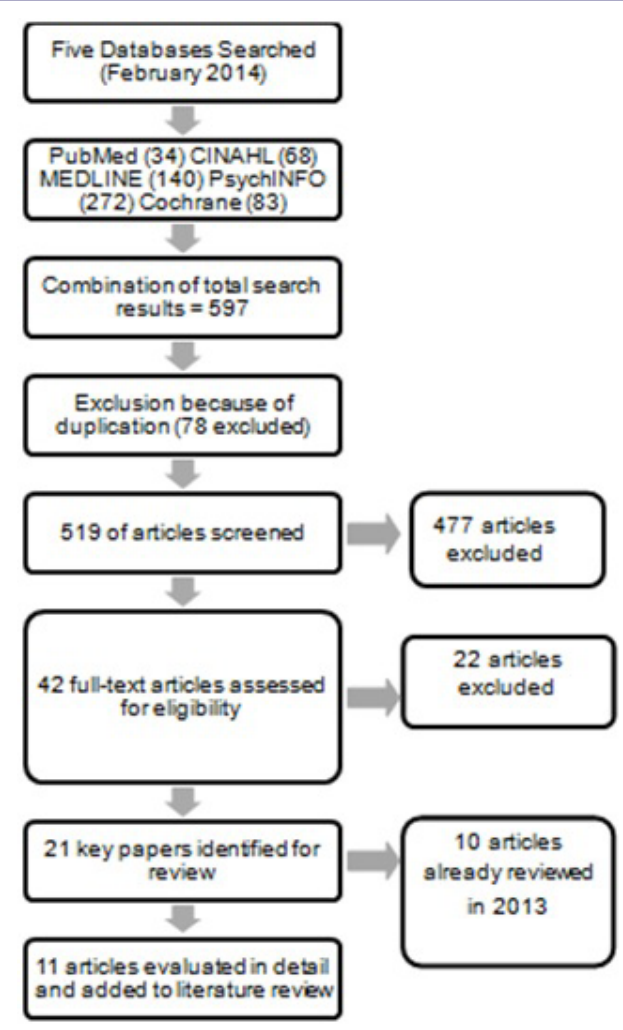

Figure 1: Flow diagram of method used for database search in 2014. 
A similar search was conducted on CINAHL using the CINAHL headings 'adjustment disorders,' 'depression, reactive,' 'generalized anxiety disorder, ' 'anxiety disorders,' 'depression,' or 'anxiety', These results were narrowed by searching with the heading 'diabetes mellitus, Type 1' as well as the results from a title search for 'Type 1 AND diabetes'. These results were then further narrowed by excluding titles containing 'type 2 AND diabetes', or 'child* or adolescence ${ }^{*}$, and then limit of articles published from 2009 was applied. Thesaurus terms in PsychINFO selected were 'dysthymic disorder', 'major depression', 'adjustment disorder', 'anxiety', 'anxiety disorder', 'depression (emotion)', 'atypical depression', 'recurrent depression,' These results were then searched with the terms 'diabetes' or 'diabetes mellitus' or titles including 'type 1 AND diabetes.' The results were similarly narrowed by excluding type 2 diabetes, child ${ }^{\star}$ and adolescence ${ }^{\star}$ from the search, and a limit was applied to exclude articles published prior to 2009. for use because many of the articles were observational studies, as such an alternative tailored approach was adopted [24]. Various approaches to quality assessment in observational studies have been used and although there is no 'gold standard', the development of content-specific assessment components is a viable recommended approach [25]. As such, individual components were chosen to highlight key factors in methodologies that may have contributed to bias (Table 1). Articles that fulfilled the requirements of a component are marked in the table with a tick and those that did not are marked with a cross. In some instances it was unclear from the study as to whether or not a criterion was met and in these instances 'unclear' was inserted.

The essential information extracted from the identified publications were: participant demographics; measurement tools; prevalence of depression; diabetes-specific and non-diabetes specific

\begin{tabular}{|c|c|c|c|c|c|}
\hline Study & Sample size & Measure of depression & $\begin{array}{c}\text { Total with depression } \\
(\%)\end{array}$ & $\begin{array}{c}\text { Men with depression } \\
\text { (\% of men) }\end{array}$ & $\begin{array}{l}\text { Women with depression } \\
\text { (\% of women) }\end{array}$ \\
\hline Melin et al., & 292 & HADS score $\geq 8$ & $30(10 \%)$ & $16(10 \%)$ & $14(11 \%)$ \\
\hline Maia et al., & 100 with T1 DM (200 total) & MINI; "current depression" & $9(9 \%)$ & - & - \\
\hline van Bastelaar et al., & 280 with T1 DM (627 total) & CES - D & $41(15 \%)$ & - & - \\
\hline Collins et al., & 296 with T1 DM (1456 total) & HADS score $\geq 8$ & $60(20 \%)$ & $34(23 \%)$ & $26(21 \%)$ \\
\hline Ahola et al., & 1266 & BDI score $\geq 16$ & $214(17 \%)$ & $78(14 \%)$ & $136(20 \%)$ \\
\hline \multirow[t]{3}{*}{ Pouwer et al., } & 379 with T1 DM (722 total) & WHO-5 $\leq 28$ & $51(13 \%)$ & $18(11 \%)$ & $33(16 \%)$ \\
\hline & 311 & CES-D score $\geq 16$ & $93(30 \%)$ & $33(25 \%)$ & $60(33 \%)$ \\
\hline & $142^{+}$ & $\begin{array}{c}\text { CIDI - major depressive } \\
\text { disorder }\end{array}$ & $11(8 \%)$ & $5(8 \%)$ & $6(8 \%)$ \\
\hline \multirow[t]{2}{*}{ Gendelman et al., } & 458 with T1 DM) (1004 total) & BDI score $\geq 14$ & $-17 \%$ & $-14 \%$ & $-20 \%$ \\
\hline & & $\begin{array}{l}\text { BDI score } \geq 14 \text { or } \\
\text { antidepressant use }\end{array}$ & $(32 \%)^{* \neq}$ & $(26 \%)^{*}$ & $-38 \%$ \\
\hline Ruddock et al., & 111 with T1 DM (211 total) & HADS score $\geq 8$ & $16(14 \%)$ & - & - \\
\hline Manarte \& Dias & 106 with T1 DM & Hamilton score $\geq 7$ & $57(54 \%)$ & & \\
\hline \multirow[t]{2}{*}{ Lloyd et al., } & 264 with T1 DM & CES-D score $\geq 16$ & $47(18 \%)$ & $14(11 \%)$ & $33(25 \%)$ \\
\hline & & BDI score $\geq 16$ & $35(14 \%)$ & $21(16 \%)$ & $14(11 \%)$ \\
\hline Hirai et al., & 484 & CES-D score $\geq 16$ & $183(38 \%)$ & - & - \\
\hline Grant et al., & $\begin{array}{l}225 \text { with T1 DM; non pump } \\
\text { group ( } 775 \text { total) }\end{array}$ & $\begin{array}{l}\text { Medical records and } \\
\text { psychotropic use }\end{array}$ & $-37 \%$ & - & - \\
\hline Tanebaum \& Gonzalez & 34 & $\begin{array}{l}\text { Medical history of either past, } \\
\text { current or recurrent major } \\
\text { depressive disorder }\end{array}$ & $15(44 \%)$ & - & - \\
\hline \multirow[t]{2}{*}{ Hopkins et al., } & 484 & HADS score $\geq 8$ baseline & $(21 \%)^{\alpha}$ & - & - \\
\hline & & $\begin{array}{c}\text { HADS } \geq 8 \text { after DAFNE } \\
\text { education }\end{array}$ & $(15 \%)^{\alpha}$ & - & - \\
\hline Speight et al., & 1376 with T1 DM (3338 total) & $P H Q-9$ score $\geq 10$ & $-22 \%$ & - & - \\
\hline
\end{tabular}

Table 1: Prevalence of depression in adults with type 1 diabetes mellitus.

Centre for Epidemiologic Studies Depression Scale (CES-D): Beck Depression Inventory; Composite International Diagnostic Interview (CIDI); Hospital Anxiety Depression Scale (HADS); Mini International Neuropsychiatric Interview (MINI); Patient Health Questionnaire (PHQ-9); World Health Organisation (WHO-5);

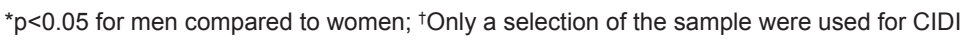

¥Gendelman et al., reported that adults with Type 1 DM were twice as likely to be depressed than non-diabetic participants

aHopkins et al., found a significant improvement in HADS score for depression after DAFNE education programs compared to baseline levels

\section{Data extraction and review}

Eligibility assessment was performed by a single reviewer. An initial screen of titles was performed, and then abstracts. Articles were excluded if they did not meet the eligibility criteria outlined in the study protocol. The remaining papers were then examined in closer detail and excluded if not all inclusion criteria were met, or if judged as having a high risk of bias. A tool for assessing bias was not suitable risk and protective factors for depression or anxiety in people with Type $1 \mathrm{DM}$; outcomes for Type $1 \mathrm{DM}$ management and complications; and relevant qualitative data.

To calculate the average percentage prevalence of depression and anxiety reported by studies overall, percentage data from the articles that reported this finding were added and then divided by the total number included. For studies with more than one measure used and 
Citation: Subasinghe SS, Bongetti E, O'Brien CL, Silberberg C, Ward G, et al. (2015) A Review of the Prevalence and Associations of Depression and Anxiety in Type 1 Diabetes Mellitus. J Diabetes Metab Disord 2: 007.

- Page 4 of $11 \bullet$

\begin{tabular}{|c|c|c|c|c|c|c|c|c|c|c|}
\hline Study & $\mathbf{N}$ & $\begin{array}{c}\text { Number } \\
\text { with Type } \\
1 \mathrm{DM}\end{array}$ & $\begin{array}{c}\text { Mean } \\
\text { age } \\
\text { (years) }\end{array}$ & $\begin{array}{l}\text { Type } 1 \\
\text { DM mean } \\
\text { duration } \\
\text { (years) }\end{array}$ & $\begin{array}{c}\text { Mean } \\
\text { HbA1c } \\
(\%)\end{array}$ & Setting & Country & Selection & $\begin{array}{l}\text { Exclusion criteria } \\
\text { for selection }\end{array}$ & $\begin{array}{c}\text { Outcome measures and } \\
\text { assessment }\end{array}$ \\
\hline $\begin{array}{l}\text { Melin et } \\
\text { al., }\end{array}$ & 292 & 292 & 41 & 21.1 & 8.3 & $\begin{array}{l}\text { Hospital } \\
\text { outpatient } \\
\text { clinic }\end{array}$ & Sweden & $\begin{array}{l}\text { Patients selected } \\
\text { from clinic during } \\
2009 \text { by specialist } \\
\text { nurse or physician. }\end{array}$ & $\begin{array}{l}\text { Severe comor- } \\
\text { bidities (cancer, } \\
\text { hepatic failure, } \\
\text { end-stage renal } \\
\text { disease, etc), } \\
\text { severe psychiatric } \\
\text { illness (severe } \\
\text { psychosis, severe } \\
\text { substance abuse, } \\
\text { etc) }\end{array}$ & $\begin{array}{l}\text { Depression and anxiety } \\
\text { measured with HADS, } \\
\text { with cut-off score of } \geq 8 \text {. } \\
\text { HbA1c was performed } \\
\text { in the clinic at time } \\
\text { as HADS. Data was } \\
\text { collected from medical } \\
\text { records about diabetes } \\
\text { complications. }\end{array}$ \\
\hline $\begin{array}{l}\text { Maia et } \\
\text { al., }\end{array}$ & 200 & 100 & 57.62 & - & - & $\begin{array}{l}\text { Community } \\
\text { outpatient } \\
\text { clinic }\end{array}$ & Brazil & $\begin{array}{l}\text { Patients recruited } \\
\text { from clinic who } \\
\text { had been already } \\
\text { diagnosed with either } \\
\text { Type } 1 \text { DM or Type } \\
2 \text { DM. }\end{array}$ & Not specified & $\begin{array}{l}\text { The MINI was used to } \\
\text { assess each participant } \\
\text { to screen for a range of } \\
\text { psychiatric disorders. }\end{array}$ \\
\hline $\begin{array}{c}\text { van } \\
\text { Bastelaar } \\
\text { et al., }\end{array}$ & 627 & 280 & 44 & 22 & 7.8 & $\begin{array}{l}\text { Hospital } \\
\text { outpatient } \\
\text { clinic }\end{array}$ & Netherlands & $\begin{array}{l}\text { Data about patients } \\
\text { was taken from an- } \\
\text { other study (1) where } \\
\text { people with Type } 1 \\
\text { DM and Type } 2 \text { DM } \\
\text { were randomly sam- } \\
\text { pled from } 3 \text { diabetes } \\
\text { clinics. }\end{array}$ & $\begin{array}{l}\text { History of suicide, } \\
\text { previous admis- } \\
\text { sion for depres- } \\
\text { sion, diagnosis of } \\
\text { schizophrenia }\end{array}$ & $\begin{array}{l}\text { HbA1c was recorded } \\
\text { from most recent medical } \\
\text { records. Depressive } \\
\text { symptoms were mea- } \\
\text { sured with CES-D scale } \\
\text { with a cut-off score of } \geq \\
16 \text {. PAID score (problem } \\
\text { areas in diabetes) }\end{array}$ \\
\hline $\begin{array}{l}\text { Sacco et } \\
\text { al., }\end{array}$ & 124 & 32 & 43.4 & - & 8.3 & $\begin{array}{l}\text { Community } \\
\text { outpatient } \\
\text { clinic }\end{array}$ & USA & $\begin{array}{l}\text { Patients selected } \\
\text { from a specialist dia- } \\
\text { betes outpatient clinic } \\
\text { from waiting room or } \\
\text { by telephone. }\end{array}$ & Not specified. & $\begin{array}{l}\text { Depression assessed } \\
\text { with Patient Health } \\
\text { Questionnaire (PHQ-9). } \\
\text { No cut-off score was } \\
\text { specified by authors. } \\
\text { HbA1c was obtained from } \\
\text { medical records. } 90 \% \text { of } \\
\text { values were taken within } \\
60 \text { days prior to comple- } \\
\text { tion of questionnaires. }\end{array}$ \\
\hline Bot et al., & 642 & 277 & 43.9 & 22.1 & 7.8 & $\begin{array}{l}\text { Hospital } \\
\text { outpatient } \\
\text { clinic }\end{array}$ & Netherlands & $\begin{array}{l}\text { Data about patients } \\
\text { was taken from an- } \\
\text { other study (1) where } \\
\text { people with Type } 1 \\
\text { DM and Type } 2 \text { DM } \\
\text { were randomly sam- } \\
\text { pled from } 3 \text { diabetes } \\
\text { clinics. }\end{array}$ & Not specified & $\begin{array}{l}\text { Depressive symptoms as- } \\
\text { sessed with PHQ-9. Each } \\
\text { symptom was assessed } \\
\text { independently and } \\
\text { considered significant if } \\
\text { scored as being present } \\
\text { 'nearly all the time' or } \\
\text { 'more than half the time.' } \\
\text { HbA1c was taken from } \\
\text { medical records. }\end{array}$ \\
\hline $\begin{array}{l}\text { Ahola et } \\
\text { al., }\end{array}$ & 1226 & 1226 & 44.9 & 27 & 8 & $\begin{array}{l}\text { Hospital and } \\
\text { community } \\
\text { outpatient } \\
\text { clinics }\end{array}$ & Finland & $\begin{array}{l}\text { Patients were re- } \\
\text { cruited for FinnDiane } \\
\text { study from hospitals } \\
\text { and primary health } \\
\text { care centers. All pa- } \\
\text { tients with Type1 DM } \\
\text { at diabetic and renal } \\
\text { clinics and dialysis } \\
\text { units were asked to } \\
\text { participate. Response } \\
\text { rate } 78 \% \text {. }\end{array}$ & $\begin{array}{l}\text { Patients with an } \\
\text { age at diagnosis } \\
>35 \text { years or who } \\
\text { had not had insulin } \\
\text { therapy initiated } \\
\text { within } 1 \text { year after } \\
\text { diagnosis were } \\
\text { excluded. }\end{array}$ & $\begin{array}{l}\text { Depression assessed } \\
\text { with the Beck inventory } \\
\text { score (BDI) cut-off } \geq 16 \text {, } \\
\text { or current use of an- } \\
\text { ti-depressants. Diabetic } \\
\text { complications obtained } \\
\text { from medical records. }\end{array}$ \\
\hline $\begin{array}{l}\text { Collins et } \\
\text { al., }\end{array}$ & 1456 & 296 & - & - & - & $\begin{array}{l}\text { Hospital and } \\
\text { community } \\
\text { outpatient } \\
\text { clinics }\end{array}$ & Ireland & $\begin{array}{l}\text { Patients from hospital } \\
\text { and general practice } \\
\text { settings were mailed } \\
\text { surveys to complete } \\
\text { and return. }\end{array}$ & Not specified & $\begin{array}{l}\text { Depression and anxiety } \\
\text { were assessed with } \\
\text { HADS and cut-off points } \\
\text { for mild (8-10), moderate } \\
\text { (11-14) and severe (15- } \\
\text { 21) were used. }\end{array}$ \\
\hline $\begin{array}{l}\text { Pouwer et } \\
\text { al., }\end{array}$ & 722 & 359 & 43 & 22 & 7.8 & $\begin{array}{l}\text { Hospital } \\
\text { outpatient } \\
\text { clinic }\end{array}$ & Netherlands & $\begin{array}{l}\text { Data about patients } \\
\text { was taken from a } \\
\text { larger study (1) where } \\
\text { people with Type } 1 \\
\text { DM and Type } 2 \text { DM } \\
\text { were randomly sam- } \\
\text { pled from } 3 \text { diabetes } \\
\text { clinics. }\end{array}$ & Not specified & $\begin{array}{l}\text { Depressive symptoms } \\
\text { self-reported on WHO- } 5 \text {. } \\
\text { Scores } \leq 28 \text { are sugges- } \\
\text { tive of depression. CES-D } \\
\text { scale also used with a } \\
\text { cut-off of } \geq 16 \text {. WHO } \\
\text { Composite International } \\
\text { Diagnostic Interview } \\
\text { (CIDI) also used to deter- } \\
\text { mine the presence of a } \\
\text { mood disorder in the past } \\
12 \text { months. }\end{array}$ \\
\hline
\end{tabular}


Citation: Subasinghe SS, Bongetti E, O'Brien CL, Silberberg C, Ward G, et al. (2015) A Review of the Prevalence and Associations of Depression and Anxiety in Type 1 Diabetes Mellitus. J Diabetes Metab Disord 2: 007.

- Page 5 of $11 \bullet$

\begin{tabular}{|c|c|c|c|c|c|c|c|c|c|c|}
\hline $\begin{array}{c}\text { Gendle- } \\
\text { man et } \\
\text { al., }\end{array}$ & 1004 & 458 & 43.6 & 29.4 & 7.9 & $\begin{array}{l}\text { Community } \\
\text { Outpatient } \\
\text { clinic }\end{array}$ & USA & $\begin{array}{l}\text { Patients were from } \\
\text { another study (2) } \\
\text { which recruited } \\
\text { participants from the } \\
\text { community. }\end{array}$ & $\begin{array}{l}\text { Excluded if not } \\
\text { diagnosed when }< \\
30 \text { years old or not } \\
\text { treated with insulin } \\
\text { within } 1 \text { year of } \\
\text { diagnosis }\end{array}$ & $\begin{array}{l}\text { BDI-II was used with a } \\
\text { cut-off of } \geq 14 \text {. Anti-de- } \\
\text { pressant medication use } \\
\text { was also reported by } \\
\text { patients and verified by } \\
\text { researchers. }\end{array}$ \\
\hline $\begin{array}{l}\text { Shaban et } \\
\text { al., }\end{array}$ & 259 & 259 & 40 & 18 & 8.8 & $\begin{array}{l}\text { Hospital } \\
\text { outpatient } \\
\text { clinic }\end{array}$ & $\begin{array}{l}\text { United King- } \\
\text { dom }\end{array}$ & $\begin{array}{l}\text { Patients with Type } 1 \\
\text { DM were recruited } \\
\text { from outpatient } \\
\text { clinics. }\end{array}$ & $\begin{array}{l}\text { If duration of Type } \\
1 \mathrm{DM}<1 \text { year, } \\
\text { not on flexible } \\
\text { dose adjustment } \\
\text { regimen }\end{array}$ & $\begin{array}{l}\text { HADS was used to } \\
\text { assess anxiety and de- } \\
\text { pression. A cut-off score } \\
\text { was not specified. HbA1C } \\
\text { within one month prior to } \\
\text { questionnaire completion } \\
\text { was recorded from medi- } \\
\text { cal records. }\end{array}$ \\
\hline $\begin{array}{l}\text { Ruddock } \\
\text { et al., }\end{array}$ & 211 & 111 & 44.9 & 23.1 & 7.9 & $\begin{array}{l}\text { Hospital } \\
\text { outpatient } \\
\text { clinic }\end{array}$ & $\begin{array}{l}\text { United King- } \\
\text { dom }\end{array}$ & $\begin{array}{l}\text { Patients from a dia- } \\
\text { betes outpatient clinic } \\
\text { were invited by mail } \\
\text { to participate. }\end{array}$ & $\begin{array}{l}\text { Excluded if }<18 \\
\text { years old, severe } \\
\text { comorbidities, cog- } \\
\text { nitive or language } \\
\text { deficits. }\end{array}$ & $\begin{array}{l}\text { HADS with a score of } \geq \\
8 \text { was used to indicate } \\
\text { either depression or } \\
\text { anxiety symptoms. }\end{array}$ \\
\hline $\begin{array}{l}\text { Lloyd et } \\
\text { al., }\end{array}$ & 264 & 264 & 45 & 36.7 & 7.5 & $\begin{array}{l}\text { Hospital } \\
\text { outpatient } \\
\text { clinic }\end{array}$ & $\begin{array}{l}\text { United King- } \\
\text { dom }\end{array}$ & $\begin{array}{l}\text { Data from the Pitts- } \\
\text { burgh Epidemiology } \\
\text { of Diabetes Compli- } \\
\text { cations was used. }\end{array}$ & $\begin{array}{l}\text { Excluded if not } \\
\text { diagnosed before } \\
\text { the age of } 17 \text { or } \\
\text { not seen within a } \\
\text { year of diagnosis } \\
\text { at the Children's } \\
\text { Hospitals of } \\
\text { Pittsburgh in 1950- } \\
1980\end{array}$ & $\begin{array}{l}\text { BDI scores } \geq 16 \text { and } \\
\text { CES-D scores } \geq 16 \text { were } \\
\text { used to indicate the } \\
\text { presence of depressive } \\
\text { symptoms. Details about } \\
\text { diabetes complications } \\
\text { was retrieved from med- } \\
\text { ical files. }\end{array}$ \\
\hline $\begin{array}{l}\text { Reichel et } \\
\text { al., }\end{array}$ & 52 & 52 & 41.4 & 20.9 & 7.16 & $\begin{array}{l}\text { Not spec- } \\
\text { ified }\end{array}$ & Germany & $\begin{array}{l}\text { Sample selection not } \\
\text { specified }\end{array}$ & Not specified & $\begin{array}{l}\text { HADS was used to as- } \\
\text { sess both depression and } \\
\text { anxiety. Cut-off scores } \\
\text { were not used although } \\
\text { the authors refer to 'mild' } \\
\text { and 'severe' depression } \\
\text { and anxiety symptoms } \\
\text { which the HADS has } \\
\text { recommended scores } \\
\text { for. Participants were } \\
\text { all using a continuous } \\
\text { subcutaneous insulin } \\
\text { infusion pump so their di- } \\
\text { abetes had been clinically } \\
\text { diagnosed. }\end{array}$ \\
\hline $\begin{array}{l}\text { Rasmus- } \\
\text { sen et al., }\end{array}$ & 20 & 20 & - & - & - & $\begin{array}{l}\text { Hospital } \\
\text { outpatient } \\
\text { clinic }\end{array}$ & Australia & $\begin{array}{l}\text { Patients recruited } \\
\text { from a flyer in the city, } \\
\text { a hospital outpatient } \\
\text { clinic and support } \\
\text { group website }\end{array}$ & $\begin{array}{l}\text { Excluded if not } \\
\text { between the ages } \\
\text { of } 18-38 \text { years. }\end{array}$ & $\begin{array}{l}\text { This was a qualitative } \\
\text { study that assessed sig- } \\
\text { nificant transition stages } \\
\text { in their lives and their } \\
\text { main concerns living with } \\
\text { diabetes. }\end{array}$ \\
\hline $\begin{array}{c}\text { Tane- } \\
\text { baum\& } \\
\text { Gonzalez }\end{array}$ & 34 & 34 & 43.45 & - & 7.96 & $\begin{array}{l}\text { Hospital } \\
\text { outpatient } \\
\text { clinic }\end{array}$ & USA & $\begin{array}{l}\text { Participants were } \\
\text { recruited via } \\
\text { direct referrals, clinic } \\
\text { screenings, flyers. }\end{array}$ & None specified & $\begin{array}{l}\text { Semi-structured } \\
\text { interviews were used to } \\
\text { assess for the presence } \\
\text { of depressive symptoms } \\
\text { and major depressive } \\
\text { disorder. }\end{array}$ \\
\hline
\end{tabular}




\begin{tabular}{|c|c|c|c|c|c|c|c|c|c|c|}
\hline & & & & & & & & & & $\begin{array}{l}\text { The mongomery-asberg } \\
\text { depression rating scale } \\
\text { was used to evaluate } \\
\text { severity of depressive } \\
\text { symptoms. Participants } \\
\text { also completed CES-D. } \\
\text { Data was collected from } \\
\text { previous visits about } \\
\text { clinical assessments and } \\
\text { HbA1c }\end{array}$ \\
\hline $\begin{array}{l}\text { Hopkins } \\
\text { et al., }\end{array}$ & 639 & 639 & 41.5 & 13.7 & 8.51 & $\begin{array}{l}\text { Not spec- } \\
\text { ified }\end{array}$ & $\begin{array}{l}\text { United King- } \\
\text { dom }\end{array}$ & $\begin{array}{l}\text { Retrospective study } \\
\text { on patients who } \\
\text { attended a DAFNE } \\
\text { education session }\end{array}$ & $\begin{array}{l}\text { Only those with } \\
\text { follow-up data after } \\
\text { DAFNE course } \\
\text { were included } \\
\text { in the analysis. } \\
\text { These partici- } \\
\text { pants were older } \\
\text { and had lower } \\
\text { mean HbA1c\% } \\
\text { compared to those } \\
\text { who did not have } \\
\text { follow-up data. }\end{array}$ & $\begin{array}{l}\text { Data was extracted from } \\
\text { records collected by } 31 \\
\text { centres in the UK con- } \\
\text { ducted DAFNE courses } \\
\text { about HbA1c and HADS } \\
\text { at baseline and during a } \\
12 \text { month follow up period } \\
\text { after the DAFNE course. } \\
\text { HADS cut-off score } \geq 8 \\
\text { was used to assess both } \\
\text { depression and anxiety. }\end{array}$ \\
\hline $\begin{array}{l}\text { Aberle et } \\
\text { al., }\end{array}$ & 51 & 51 & 36.5 & 19 & 7.1 & $\begin{array}{l}\text { Hospital } \\
\text { Outpatient } \\
\text { clinic }\end{array}$ & Germany & $\begin{array}{l}\text { All patients attending } \\
\text { a German diabetes } \\
\text { centre who had Type } \\
\text { 1DM }\end{array}$ & None specified & $\begin{array}{l}\text { Diabetes was diagnosed } \\
\text { previously clinically. } \\
\text { HbA1c was measured } \\
\text { once consent to partic- } \\
\text { ipate was given. The } \\
\text { German version of the } \\
\text { self-rating depression } \\
\text { scale was used to assess } \\
\text { for the presence of } \\
\text { depressive symptoms. } \\
\text { Other psychological fac- } \\
\text { tors including self-efficacy } \\
\text { were measured. }\end{array}$ \\
\hline $\begin{array}{l}\text { Speight } \\
\text { et al., }\end{array}$ & 3338 & 1376 & 42 & 15.2 & 7.8 & $\begin{array}{l}\text { Community } \\
\text { Sample }\end{array}$ & Australia & $\begin{array}{l}\text { A random sample } \\
\text { registered with the } \\
\text { National Diabetes } \\
\text { Service Scheme } \\
\text { (NDSS) who were } \\
\text { contacted by mail }\end{array}$ & Not reported & $\begin{array}{l}\text { Depression was mea- } \\
\text { sured with the PHQ-9 } \\
\text { with cut-off scores of } \\
10 \text { and } 15 \text { for moderate } \\
\text { and severe depression } \\
\text { respectively. Anxiety was } \\
\text { assessed with the GAD-7 } \\
\text { with cut-off scores of } 10 \\
\text { and } 15 \text { for moderate and } \\
\text { severe anxiety respec- } \\
\text { tively. Diabetes-distress } \\
\text { was also assessed with } \\
\text { the PAID scale. }\end{array}$ \\
\hline
\end{tabular}

Table 2: Comparison of participant demographics and methods between studies.

Centre for Epidemiologic Studies Depression Scale (CES-D): Beck Depression Inventory; Composite International Diagnostic Interview (CIDI); Dose Adjustment for Normal Eating (DAFNE); GAD-7 (Generalised Anxiety Disorder scale); Hospital Anxiety Depression Scale (HADS); Mini International Neuropsychiatric Interview (MINI); Problem Areas in Diabetes (PAID); Patient Health Questionnaire (PHQ-9); World Health Organisation (WHO-5)

therefore more than one percentage value the average of that study's results was used.

\section{Results and Discussion}

Between September and October 2013234 articles were identified as being potentially relevant. Through a process of systematic exclusion, ten key papers were eventually identified and summarized (Figure 1).

In the literature search conducted in February 2014 (Figure 1) 597 articles were identified; 78 were duplicates and deleted and 454 were excluded on the basis of title and abstract.

\section{Comparison of study designs}

There was variation in research design and methods as shown in table 2. Most studies used self-report scales to measure depression and anxiety, with only two studies opting for structured diagnostic interviews [26]. Two studies included qualitative data about experiences of people living with Type $1 \mathrm{DM}[26,27]$.

There was significant variation in sample size between studies (20-1456 participants). Some studies had mixed samples of Type 1 DM and Type 2 DM however all papers in this review specified the number of participants who had Type $1 \mathrm{DM}$. Two studies were conducted in Australia [8,27] which may limit the degree to which this data can be generalized to our population.

Two studies used the same samples from a larger study conducted in the Netherlands $[5,14]$. Therefore the data included from these in this review may over-represent this sample. The age of participants between studies is relatively comparable and ranges from 37 to 58, however not all included mean ages in their results $[4,27]$. There was some variation in the duration of diabetes ( 9.3 - 36.7 years) although five studies did not publish this data.

\section{Assessment of risk of bias}

Results from an assessment of individual components relevant to risk of bias within studies are shown in table 3. Given that all studies were observational rather than randomized controlled trials, there is an inherent risk for bias within all of the studies reviewed [28], however this does not increase bias across studies. 
Citation: Subasinghe SS, Bongetti E, O'Brien CL, Silberberg C, Ward G, et al. (2015) A Review of the Prevalence and Associations of Depression and Anxiety in Type 1 Diabetes Mellitus. J Diabetes Metab Disord 2: 007.

- Page 7 of $11 \bullet$

\begin{tabular}{|c|c|c|c|c|c|c|c|c|c|c|c|c|}
\hline \multirow{9}{*}{ 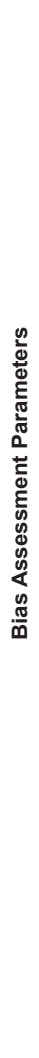 } & Study & $\begin{array}{l}\text { Melin } \\
\text { et al., }\end{array}$ & $\begin{array}{l}\text { Maia et } \\
\text { al., }\end{array}$ & $\begin{array}{l}\text { van } \\
\text { Bastelaar } \\
\text { et al., }\end{array}$ & $\begin{array}{l}\text { Sacco } \\
\text { et al., }\end{array}$ & $\begin{array}{l}\text { Bot } \\
\text { et al., }\end{array}$ & $\begin{array}{l}\text { Ahola } \\
\text { et al., }\end{array}$ & $\begin{array}{l}\text { Collins } \\
\text { et al., }\end{array}$ & $\begin{array}{l}\text { Pouwer } \\
\text { et al., }\end{array}$ & $\begin{array}{l}\text { Gendleman } \\
\text { et al., }\end{array}$ & $\begin{array}{l}\text { Shaban } \\
\text { et al., }\end{array}$ & $\begin{array}{l}\text { Ruddock } \\
\text { et al., }\end{array}$ \\
\hline & $\begin{array}{l}\text { Longitudinal Design } \\
\text { Were participants followed up over time? }\end{array}$ & $x$ & $x$ & $x$ & $x$ & $\checkmark$ & $x$ & $x$ & $x$ & $x$ & $x$ & $x$ \\
\hline & $\begin{array}{c}\text { Selection unbiased } \\
\text { Was selection clearly described and not } \\
\text { influenced by history of mental illness } \\
\text { (unless ongoing illness severe in nature)? }\end{array}$ & $\checkmark$ & Unclear & $\checkmark$ & $\checkmark$ & $\checkmark$ & $\checkmark$ & Unclear & $\checkmark$ & $\checkmark$ & Unclear & $\checkmark$ \\
\hline & $\begin{array}{c}\text { Appropriately focussed sample } \\
\text { Did the sample consist of Type 1DM } \\
\text { only? Or was there a clear distinction in } \\
\text { methods and results between Type } 1 \text { DM } \\
\text { and Type } 2 \text { DM participants? or clearly } \\
\text { differentiated between Type } 1 \text { DM and } \\
\text { Type } 2 \text { DM }\end{array}$ & $\checkmark$ & $\checkmark$ & $\checkmark$ & $\checkmark$ & $\checkmark$ & $\checkmark$ & $\checkmark$ & $\checkmark$ & $\checkmark$ & $\checkmark$ & $\checkmark$ \\
\hline & $\begin{array}{l}\text { Relevant participant variables considered } \\
\text { Reported proportion of sample with diabe- } \\
\text { tes-related complications }\end{array}$ & $\checkmark$ & $x$ & $x$ & $x$ & $\checkmark$ & $\checkmark$ & $\checkmark$ & $\checkmark$ & $\checkmark$ & $x$ & $\checkmark$ \\
\hline & $\begin{array}{l}\text { Use of a control group } \\
\text { Was a control group of people without } \\
\text { diabetes included? }\end{array}$ & $x$ & $x$ & $x$ & $x$ & $x$ & $x$ & $x$ & $x$ & $\checkmark$ & $x$ & $x$ \\
\hline & $\begin{array}{l}\text { Validity of scales } \\
\text { Were validated measures of depression } \\
\text { and anxiety used? }\end{array}$ & $\checkmark$ & $\checkmark$ & $\checkmark$ & $\checkmark$ & $\checkmark$ & $\checkmark$ & $\checkmark$ & $\checkmark$ & $\checkmark$ & $\checkmark$ & $\checkmark$ \\
\hline & $\begin{array}{c}\text { Psychotropic use } \\
\text { Was data collected data on current } \\
\text { psychotropic use at time of study and } \\
\text { included in analyses? }\end{array}$ & $\checkmark$ & $\checkmark$ & $\checkmark$ & $x$ & $\checkmark$ & $\checkmark$ & $x$ & $x$ & $\checkmark$ & $x$ & $x$ \\
\hline & $\begin{array}{l}\text { Clinical psychological assessmentWas } \\
\text { clinical assessment used to either assess } \\
\text { psychological distress or verify the validity } \\
\text { of psychological assessment scales? }\end{array}$ & $x$ & $\checkmark$ & $x$ & $x$ & $x$ & $x$ & $x$ & $\checkmark$ & $x$ & $x$ & $x$ \\
\hline
\end{tabular}

Type 1 DM: Type 1 Diabetes Mellitus; Type 2 DM: Type 2 Diabetes Mellitus

\begin{tabular}{|c|c|c|c|c|c|c|c|c|c|c|}
\hline \multirow{9}{*}{ 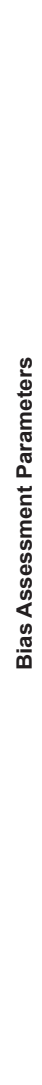 } & Study & $\begin{array}{l}\text { Manarte \& } \\
\text { Dias, }\end{array}$ & $\begin{array}{l}\text { Lloyd } \\
\text { et al., }\end{array}$ & $\begin{array}{l}\text { Hirai et } \\
\text { al., }\end{array}$ & $\begin{array}{l}\text { Reichel et } \\
\text { al., }\end{array}$ & $\begin{array}{l}\text { Rasmussen } \\
\text { et al., }\end{array}$ & $\begin{array}{l}\text { Tanebaum } \\
\text { \& Gonza- } \\
\text { lez }\end{array}$ & $\begin{array}{l}\text { Hopkins } \\
\text { et al., }\end{array}$ & $\begin{array}{l}\text { Aberle et } \\
\text { al., }\end{array}$ & $\begin{array}{l}\text { Speight et } \\
\text { al., }\end{array}$ \\
\hline & $\begin{array}{l}\text { Longitudinal Design } \\
\text { Were participants followed up over time? }\end{array}$ & $x$ & $x$ & $x$ & $x$ & $x$ & $x$ & $\checkmark$ & $x$ & $x$ \\
\hline & $\begin{array}{c}\text { Selection unbiased } \\
\text { Was selection clearly described and not } \\
\text { influenced by history of mental illness } \\
\text { (unless ongoing illness severe in nature)? }\end{array}$ & $\checkmark$ & $\checkmark$ & $\checkmark$ & unclear & $\checkmark$ & $\checkmark$ & $\checkmark$ & $\checkmark$ & $\checkmark$ \\
\hline & $\begin{array}{c}\text { Appropriately focussed sample } \\
\text { Did the sample consist of Type 1DM } \\
\text { only? Or was there a clear distinction in } \\
\text { methods and results between Type } 1 \mathrm{DM} \\
\text { and Type } 2 \text { DM participants? or clearly } \\
\text { differentiated between Type } 1 \text { DM and } \\
\text { Type } 2 \text { DM }\end{array}$ & $\checkmark$ & $\checkmark$ & $\checkmark$ & $\checkmark$ & $\checkmark$ & $\checkmark$ & $\checkmark$ & $\checkmark$ & $\checkmark$ \\
\hline & $\begin{array}{l}\text { Relevant participant variables considered } \\
\text { Reported proportion of sample with diabe- } \\
\text { tes-related complications }\end{array}$ & $\checkmark$ & $\checkmark$ & $\checkmark$ & $x$ & $x$ & $x$ & $\checkmark$ & $x$ & $\checkmark$ \\
\hline & $\begin{array}{c}\text { Use of a control group } \\
\text { Was a control group of people without } \\
\text { diabetes included? }\end{array}$ & $x$ & $x$ & $x$ & $\checkmark$ & N/A & $x$ & $\checkmark$ & $x$ & $x$ \\
\hline & $\begin{array}{l}\text { Validity of scales } \\
\text { Were validated measures of depression } \\
\text { and anxiety used? }\end{array}$ & $\checkmark$ & $\checkmark$ & $\checkmark$ & $\checkmark$ & $\checkmark$ & $\checkmark$ & $\checkmark$ & $\checkmark$ & $\checkmark$ \\
\hline & $\begin{array}{c}\text { Psychotropic use } \\
\text { Was data collected data on current } \\
\text { psychotropic use at time of study and } \\
\text { included in analyses? }\end{array}$ & $\checkmark$ & $\checkmark$ & $\checkmark$ & $x$ & N/A & $x$ & $x$ & $x$ & $\checkmark$ \\
\hline & $\begin{array}{l}\text { Clinical psychological assessment } \\
\text { Was clinical assessment used to either } \\
\text { assess psychological distress or verify } \\
\text { the validity of psychological assessment } \\
\text { scales? }\end{array}$ & $x$ & $x$ & $x$ & $x$ & N/A & $\checkmark$ & $\checkmark$ & $x$ & $x$ \\
\hline
\end{tabular}

Table 3: Assessment of risk of bias.

N/A: Not Applicable; Type 1 DM: Type 1 Diabetes Mellitus; Type 2 DM: Type 2 Diabetes Mellitus ${ }^{*}$ Qualitative study 
Most studies were cross-sectional rather than longitudinal in design. However, it was felt given the ample data available from the large number of studies in total could help balance this limitation. Importantly, all studies used validated measures for psychological assessment and all studies clearly differentiated the type of diabetes.

Not all studies included psychotropic medication use in their analyses and future study designs should aim to include this variable. Participants who are taking psychotropic medication for the treatment of depression or anxiety may not score on psychological assessment scales and this could influence results. Some studies, however, were considering the effect of depressive symptoms on glycaemic control and in these instances it was appropriate to discount those receiving treatment. Moreover, it was felt that even where psychotropic medication use could have been relevant, these study designs were otherwise sound and risk of bias was relatively low and therefore included in this review.

Risk of bias across studies in this review is notable, as shown by a relatively high degree of heterogeneity in sample size, participant characteristics, and psychological measures utilized between measures. Past studies have also commented on the need for a streamlined approach to measuring psychological variables in this population [16].

\section{Prevalence of depression}

As shown in table 1, the reported prevalence of depression between studies ranged from 9\% [29] to 54\% [30]. Although most studies used self-report as a means of psychological assessment, differences in the specific scales chosen may have contributed to the differences in prevalence rates observed.

Of the studies that used self-report the average prevalence of depression was $20 \%$ (range $10-54 \%$ ). This is higher than previously reported levels by Roy et al., who found that studies without control groups reported prevalence of depression at 12\% [10]. Gendelman et al., was the only study that included a control group of participants who did not have diabetes, and they found the prevalence of depression in people with Type $1 \mathrm{DM}$ to be $18 \%$ and even higher if people taking antidepressants were added (32\%) [31] - higher than the previously reported average prevalence of $12 \%$ in controlled studies [10].

Consistent with past research [32], studies utilising interview methods produced lower prevalence rates of depression than self-report. Maia et al., for example, found that the prevalence of 'current depression' in their cohort of 100 people with Type 1 DM was $9 \%$. This low value could be because the researchers differentiated current depression from past clinical depression and dysthymia. In addition, the fact that patients were assessed with the Mini International psychiatric Interview (MINI) may help explain why current major depression was $9 \%$ prevalence and yet overall mood disorder prevalence was high (67\%) [29].

The highest prevalence of depression was reported by Manarte et al., who used the Hamilton Score with a cut-off score of 7 and above to find that $54 \%$ of their sample had symptoms of depression [31]. This measurement tool was not used by any other study in this review and therefore no comparisons can be made on the basis of assessment. However, in their sample $75 \%$ had at least one diabetes complication and only $24 \%$ had 'well controlled' diabetes with a HbAlc below $7 \%$ [31]. Therefore it is possible that their sample is representative of people more difficult to control diabetes, and may not be applicable to

\section{Prevalence of anxiety}

Prevalence of anxiety in participants with Type $1 \mathrm{DM}$ was $25 \%$ on average (range 13 - 38\%) (Table 4). When studies that used self-report measures only were compared the average was slightly higher at $27 \%$. The significant variation in reported values reflects an inherent uncertainty within the collective literature about the overall prevalence of anxiety among people with Type 1 DM. Previous literature reviews have not assessed whether or not anxiety is higher in people with Type $1 \mathrm{DM}$ compared to the general population [10,33], therefore no comparisons can be made. However, data included in this review demonstrates that anxiety is more common in people with Type $1 \mathrm{DM}$ when compared to reported rates of anxiety in the general Australian population [34].

These high rates seem reasonable given that depression and anxiety are frequently co morbid [35]. However, it was unclear from this review whether anxiety or depression is more commonly comorbid in Type $1 \mathrm{DM}$. Maia et al., found that generalized anxiety disorder was more common than depression with a prevalence of $24 \%$ [30].

The mode of psychological assessment is an important consideration when considering these results. Three studies $[4,23,30]$ showed that at least one third of their sample had significant symptoms of anxiety. Importantly, all of these studies used the HADS measure of self-report, which may represent a propensity for higher scorings using this measure. Speight et al., used the GAD-7 as a measure of anxiety reported comparably lower rates of anxiety, and therefore it's unclear whether this discrepancy is due to differences in sample population or psychological measures.

Several factors were implicated in the risk of comorbid anxiety and Type $1 \mathrm{DM}$. Collins et al., found that the pressure to maintain glycaemic control was anxiety-provoking, as participants who perceived their blood sugar control as poor were more likely to be anxious than those who judged their control positively [4]. In contrast, Hopkin's et al., found that Dose Adjustment for Normal Eating (DAFNE) training was associated with lower scores of anxiety [36]. It is therefore possible that diabetes-specific knowledge may be an important factor in reducing anxiety.

\section{Glycaemic control and depression}

There were eight studies that examined the relationship between depression and glycaemic control.

While some data suggest that people with comorbid depression may have worse glycaemic control [14,37-39], this was not a universal finding [31,40,41], with van Bastelaar, Pouwer et al., [5] reporting that participants with depression, but without diabetes-related distress, did not have changes to their glycaemic control. Whereas those who had depression as well as distress showed sub-optimal control [5]. Hence, having insight in to whether a patient's distress is diabetes-related may be relevant to predicting impact on glycaemic control. This is in keeping with a recent report by Fisher et al., who recommend healthcare professionals understand the causes of their patient's distress when creating a tailored diabetes management plan [22].

Data also revealed that certain symptoms of depression may have more bearing on glycaemic control than others. For example Bot et al., found that sleep and appetite problems correlated with increased HbA1c [14]. Interestingly, although these individual symptoms of depression in this study were predictive of glycaemic control, participants' overall scores of self-reported of depression was not [14]. 


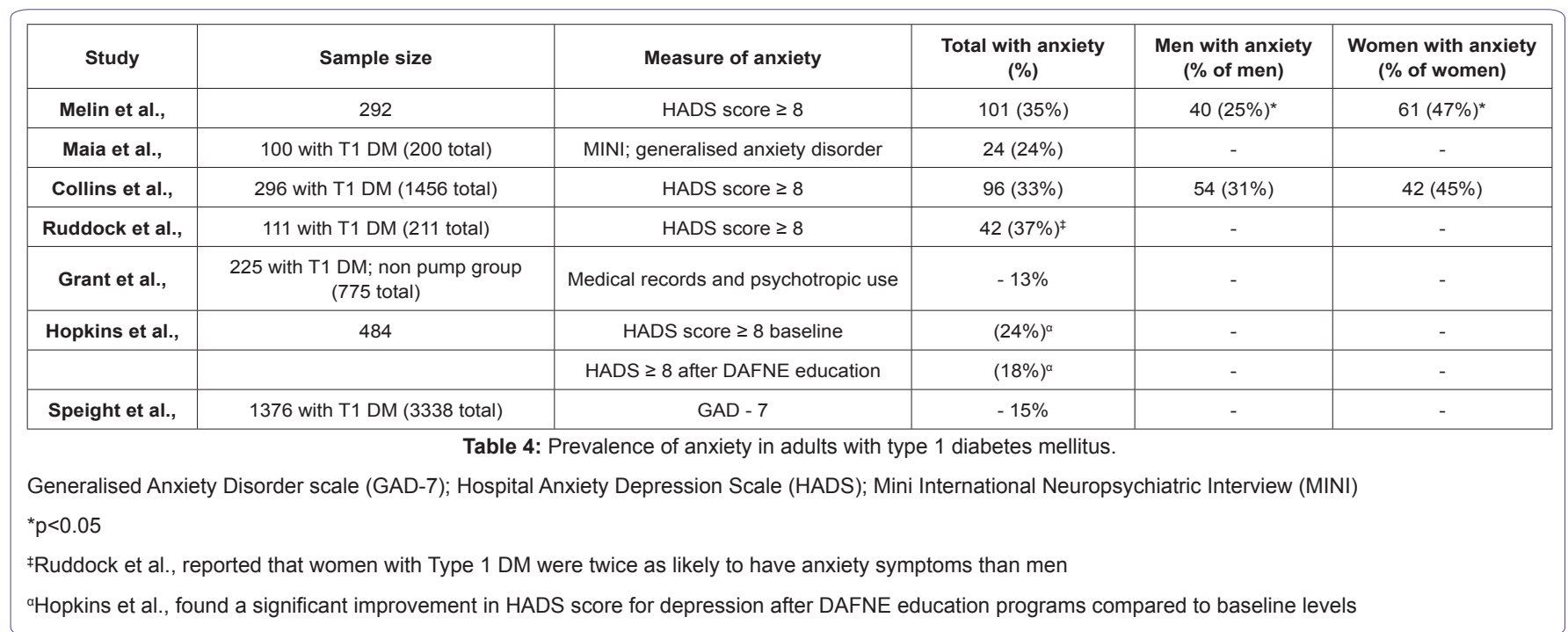

This could be because symptoms of sleep and appetite change may represent difficulties in attaining optimal blood glucose control rather than these symptoms representing the somatic symptoms of a depressive disorder.

\section{Diabetes-related complications}

Depression was associated with diabetes complications in several studies [37,28,32,42-44] however there was some conflicting evidence. There was some data that neuropathy is more common with comorbid depression [44,45]. Yet whilst Melin et al., found an association between depression and poor glycaemic control, depression did not increase the risk of diabetes complications [45].

Similarly, although Ahola et al., found that proliferative retinopathy was more common with comorbid depression [43] other studies found no association with retinopathy $[31,44]$.

Two literature reviews previously concluded that comorbid depression was associated with an increased risk of diabetes complications $[10,33]$, however none have looked exclusively at data examining people with Type $1 \mathrm{DM}$. Therefore the effect of comorbid depression on the risk of diabetes complication in people with Type 1 $\mathrm{DM}$ is not as clear-cut as those with Type $2 \mathrm{DM}$.

Individual differences that mediate the effects of comorbid depression on diabetes management: Review of the literature revealed that gender, socioeconomic status and duration of diabetes can be important risk factors for comorbid depression [43,46]. This is consistent with previous literature reviews and reflected similar trends in the general public $[42,43]$. Melin et al., found depression was more common among females compared to males [45], and Collins et al., reported anxiety was more common in females [42].

The literature also considered whether age or diabetes duration is important risk factor. Collins et al., found that older age was protective for depression in Type $1 \mathrm{DM}$, however, four other studies found increased age to be a risk factor for depression $[31,42,43,45]$. Similarly, previous literature review also found conflicting evidence for the relationship between age and depression [10].

Diabetes-specific factors such as diabetes education are important to consider in terms of their impact on psychological morbidity. Hopkins et al., found that after delivery of a structured education program to people with Type $1 \mathrm{DM}$ resulted in lower scores of depressive symptoms compared to baseline [36].

\section{Qualitative data}

Anecdotal evidence collected by Rasmussen et al., revealed that participants found their blood glucose control was influenced by significant life events, including transitions made as part of usual life development and transitions related to their diabetes $[47,48]$. It was noted that aspects of living with diabetes presented with unique additional stressors, such as the added stress of needing to manage diabetes whilst pregnant, and the development of diabetes-related complications such as depression [47].

Qualitative data from Tanebaum \& Gonzalez, 2012 reported the bi-directional effects of blood glucose and mood [27]. In particular HbA1c results caused feelings of depression and frustration, and the frequently an overlap between diabetes-related and depressive symptoms were noted. These included change in appetite, energy levels and sleep disturbance, with participants reporting it was often hard to tell if they were experiencing difficulty concentrating or sleeping because of fluctuations in their blood glucose or because of their mood. Furthermore the presence of low mood and anxiety caused further concern in some participants who worried that psychological stress would negatively impact on their glycaemic control [27].

\section{Summary and Recommendations}

This review aimed to identify and assess relevant articles that considered the prevalence of depression and anxiety among people living with Type $1 \mathrm{DM}$, and/or examined factors that may contribute to this comorbidity and the complications that can occur. An extensive literature search identified twenty-one key articles which were reviewed to provide strong evidence further supporting the notion that there is increased prevalence of comorbid depression and anxiety in Type $1 \mathrm{DM}$.

Based on the literature reviewed, our article shows that up to two in five people living with Type $1 \mathrm{DM}$ suffer from comorbid clinical depression, and one in four experience anxiety. There was variation in the exact rates reported between articles, however, which may be reflective of differences in methods or samples. Past research has also noted the significant heterogeneity of methods in research of depression in diabetes, highlighting a need for the streamlining of psychological assessment to allow for more accurate comparisons and stronger conclusions to be drawn [22]. 
In addition only seven articles measured levels of anxiety in their participants, and little is still known about complications associated with comorbid anxiety in Type $1 \mathrm{DM}$. Past reviews have also neglected to consider comorbid anxiety $[10,33]$ so it's not possible to make comparisons. More research in to the nature of anxiety in people with Type $1 \mathrm{DM}$ is warranted, especially given that depression and anxiety are usually co-occurring.

The large prevalence of anxiety and depression amongst people living with Type $1 \mathrm{DM}$ has significant implications for a person's psychological well-being, overall physical health, their diabetes care and general quality of life. For these reasons it is important to consider how evidence from these studies can inform practice. Research has shown that it is possible to effectively screen for depression in a primary care and specialist settings using simple and time-efficient two-stem questions [10]. However, given that time constraints are common in the clinical setting, it's important that specific risk factors are identified so that screening may be targeted to people at greatest risk. Review of the literature found that risk factors for the development of depression in people with Type 1 DM largely reflected the risk factors same as for the general population, such as female gender and lower socioeconomic status. Diabetes-specific risk factors were also found, including low self-efficacy regarding diabetes management and perceived poor glycaemic control. Therefore, people who have low levels of education, poor health literacy, sub-optimal glycaemic control and a history of depression and anxiety should screened on a regular basis for diabetes-distress, clinical depression and anxiety disorders.

Whilst screening is crucial, it is important for healthcare workers to have a deeper understanding of the lived experiences of people with Type 1 DM $[22,27]$. Having insight in to diabetes and non-diabetes stressors provides important context for the assessment and management of depression and anxiety, and can improve patient-physician relationships which can in turn result in improved communication and adherence [20]. This insight can be gained with clinical experience, good communication with patients, and with information gathered from qualitative studies. However, there were only two studies identified in this review that were qualitative, and most of the remaining studies relied on self-report measures.

Understanding the lived experience of Type $1 \mathrm{DM}$ is also important for the delivery of tailored psych education, specific to depression and anxiety in the context of Type $1 \mathrm{DM}$. The importance of psych education specific to people living with Type $1 \mathrm{DM}$ has been highlighted in other studies $[18,22]$, but is an area of research that requires more investigation.

\section{Limitations}

Despite an extensive and systematic literature search this study has a number of limitations. Firstly, the papers identified were mainly observational studies, and it is therefore not possible to draw conclusions about the direction of the relationship between Type $1 \mathrm{DM}$ and depression. Although this review did not identify much evidence on the biological aspects of diabetes as a risk factor for the pathogenesis of depression, it is likely that a bidirectional relationship exists.

Secondly, the assessment of depression in the studies was often by self-report, which may not reflect true diagnoses of depressive illnesses. It has been found that compared to diagnostic interview, self-report measures of depression may over-estimate the prevalence of depression [3,32]. Although self-report is an efficient and cost-effective method of assessment, diagnostic interview would increase the validity of results.

Thirdly, there was significant heterogeneity in both the methods and samples between studies. This made it difficult to make accurate comparisons between the results. Consensus within the literature on tools for assessing psychological distress within this population is needed. Ideally assessment would reconcile diabetes-distress with symptoms of depression and anxiety. Nevertheless, the diversity of samples studied also provides robustness to the collective evidence, as it demonstrates that the observation of increased depression and anxiety in people living with Type $1 \mathrm{DM}$ is a phenomenon observed across a range of demographics.

Furthermore the literature search was limited to articles published in English. Many of these studies were conducted overseas which may limit the degree to which these results can be generalized to our local population. It would be invaluable to have some research conducted within Australia to see if there are population-specific variables that need to be taken in to account.

\section{Conclusion}

The pertinence of the evidence analyzed in this review is underpinned by findings from large international studies that have shown depression results in worse health outcomes for individuals compared to any other chronic disease, including diabetes [6]. Further, the combination of depression and diabetes leads to even poorer outcomes than does the combination of diabetes and other chronic diseases. Therefore for people living with Type 1 DM, depression and anxiety are potentially the largest impediments to attaining optimal health and overall quality of life.

This review has provided evidence to support the finding that depression and anxiety are more common in people with Type $1 \mathrm{DM}$ and that it is under-diagnosed and undertreated.

Ongoing research in this area will allow for the development of tailored screening tools or assessment guidelines that can help to improve diagnosis of psychological disorders in Type 1 DM. Future research will also allow for the development of structured, evidence-based interventions to reduce psychological morbidity in this group and ultimately improve overall patient care.

\section{References}

1. Katon W, Fan MY, Unützer J, Taylor J, Pincus H, et al. (2008) Depression and diabetes: a potentially lethal combination. J Gen Intern Med 23: 1571-1575.

2. Lloyd CE (2010) Diabetes and mental health; the problem of co-morbidity. Diabet Med 27: 853-854.

3. Anderson RJ, Freedland KE, Clouse RE, Lustman PJ (2001) The prevalence of comorbid depression in adults with diabetes: a meta-analysis. Diabetes Care 24: 1069-1078.

4. Collins MM, Corcoran P, Perry IJ (2009) Anxiety and depression symptoms in patients with diabetes. Diabet Med 26: 153-161.

5. van Bastelaar KM, Pouwer F, Geelhoed-Duijvestijn PH, Tack CJ, Bazelmans E, et al. (2010) Diabetes-specific emotional distress mediates the association between depressive symptoms and glycaemic control in Type 1 and Type 2 diabetes. Diabet Med 27: 798-803.

6. Moussavi S, Chatterji S, Verdes E, Tandon A, Patel V, et al. (2007) Depression, chronic diseases, and decrements in health: results from the World Health Surveys. Lancet 370: 851-858.

7. Mathers CD, Vos ET, Stevenson CE, Begg SJ (2000) The Australian Burden of Disease Study: measuring the loss of health from diseases, injuries and risk factors. Med J Aust 172: 592-596. 
Citation: Subasinghe SS, Bongetti E, O'Brien CL, Silberberg C, Ward G, et al. (2015) A Review of the Prevalence and Associations of Depression and Anxiety in Type 1 Diabetes Mellitus. J Diabetes Metab Disord 2: 007.

8. Speight J, Browne JL, Holmes-Truscott E, Hendrieckx C, Pouwer F (2012) Diabetes MILES--Australia (management and impact for long-term empowerment and success): methods and sample characteristics of a national survey of the psychological aspects of living with type 1 or type 2 diabetes in Australian adults. BMC Public Health 12: 120.

9. Korczak DJ, Pereira S, Koulajian K, Matejcek A, Giacca A (2011) Type 1 diabetes mellitus and major depressive disorder: evidence for a biological link. Diabetologia 54: 2483-2493.

10. Roy T, Lloyd CE (2012) Epidemiology of depression and diabetes: a systematic review. J Affect Disord 142: 8-21.

11. Barnard KD, Skinner TC, Peveler R (2006) The prevalence of co-morbid depression in adults with Type 1 diabetes: systematic literature review. Diabet Med 23: 445-448.

12. Jani BD, Purves D, Barry S, Cavanagh J, McLean G, et al. (2013) Challenges and implications of routine depression screening for depression in chronic disease and multimorbidity: a cross sectional study. PLoS One 8: 74610.

13. Surwit RS, van Tilburg MA, Parekh PI, Lane JD, Feinglos MN (2005) Treatment regimen determines the relationship between depression and glycemic control. Diabetes Research and Clinical Practice 69: 78-80.

14. Rasmussen B, Ward G, Jenkins A, King SJ, Dunning T (2011) Young adults' management of Type 1 diabetes during life transitions. J Clin Nurs 20: 19811992.

15. Lloyd CE, Pambianco G, Orchard TJ (2010) Does diabetes-related distress explain the presence of depressive symptoms and/or poor self-care in individuals with Type 1 diabetes? Diabet Med 27: 234-237.

16. Fisher L, Skaff MM, Mullan JT, Arean P, Glasgow R, et al. (2008) A longitudinal study of affective and anxiety disorders, depressive affect and diabetes distress in adults with Type 2 diabetes. Diabet Med 25: 1096-1101.

17. Gendelman N, Snell-Bergeon JK, McFann K, Kinney G, Paul Wadwa R, et al. (2009) Prevalence and correlates of depression in individuals with and without type 1 diabetes. Diabetes Care 32: 575-579.

18. Aberle I, Scholz U, Bach-Kliegel B, Fischer C, Gorny M, et al. (2009) Psychological aspects in continuous subcutaneous insulin infusion: $A$ retrospective study. J Psychol 143: 147-160.

19. Rost K, Nutting P, Smith J, Coyne JC, Cooper-Patrick L, et al. (2000) The role of competing demands in the treatment provided primary care patients with major depression. Arch Fam Med 9: 150-154.

20. Ahola AJ, Groop PH (2013) Barriers to self-management of diabetes. Diabet Med 30: 413-420.

21. Fisher L, Gonzalez JS, Polonsky WH (2014) The confusing tale of depression and distress in patients with diabetes: a call for greater clarity and precision. Diabet Med 31: 764-772.

22. Ruddock S, Fosbury J, Smith A, Meadows K, Crown A (2010) Measuring psychological morbidity for diabetes commissioning: a cross-sectional survey of patients attending a secondary care diabetes clinic. Practical Diabetes International 27: 22-26.

23. Mezuk B, Eaton WW, Albrecht S, Golden SH (2008) Depression and type 2 diabetes over the lifespan: a meta-analysis. Diabetes Care 31: 2383-2390.

24. Viswanathan M, Berkman ND, Dryden DM, Hartling L (2013) Assessing risk of bias and confounding in observational studies of interventions or exposures: further development of the RTI item bank. Agency for healthcare research and quality, Methods research reports, Rockville, USA.

25. Deeks JJ, Dinnes J, D'Amico R, Sowden AJ, Sakarovitch C, et al. (2003) Evaluating non-randomised intervention studies. National Coordinating Centre for Health Technology Assessment 7: 1-179.

26. Tanenbaum ML, Gonzalez JS (2012) The influence of diabetes on a clinician-rated assessment of depression in adults with type 1 diabetes. Diabetes Educ 38: 695-704

27. Rasmussen B, Ward G, Jenkins A, King SJ, Dunning T (2011) Young adults management of Type 1 diabetes during life transitions. J Clin Nurs 20: 1981 1992.
28. Tacconelli E (2010) Systematic reviews: CRD's guidance for undertaking reviews in health care. The Lancet Infectious Diseases 10: 226.

29. Maia AC, Braga Ade A, Brouwers A, Nardi AE, Oliveira e Silva AC (2012) Prevalence of psychiatric disorders in patients with diabetes types 1 and 2 . Compr Psychiatry 53: 1169-1173.

30. Manarte LF, Dias S, Góis C, Boavida JM (2010) Independent factors associated with depression in type 1 diabetes mellitus. Acta Diabetol 47: 201-207.

31. Gendelman N, Snell-Bergeon JK, McFann K, Kinney G, Paul Wadwa R, et al. (2009) Prevalence and correlates of depression in individuals with and without type 1 diabetes. Diabetes Care 32: 575-579.

32. Pouwer F, Wijnhoven HA, Ujcic-Voortman JK, de Wit M, Schram MT, et al. (2013) Ethnic aspects of emotional distress in patients with diabetes--the Amsterdam Health Monitor Study. Diabet Med 30: 25-31.

33. Sane (2011) Anxiety Disorders. Sane Australia.

34. Schoevers RA, van HL, Koppelmans V, Kool S, Dekker JJ (2008) Managing the patient with co-morbid depression and an anxiety disorder. Drugs 68: 1621-1634.

35. Hopkins D, Lawrence I, Mansell P, Thompson G, Amiel S, et al. (2012) Improved biomedical and psychological outcomes 1 year after structured education in flexible insulin therapy for people with type 1 diabetes: the UK. DAFNE experience. Diabetes Care 35: 1638-1642.

36. Reichel A, Schwarz J, Schulze J, Licinio J, Wong ML, et al. (2005) Depression and anxiety symptoms in diabetic patients on Continuous Subcutaneous Insulin Infusion (CSII). Mol Psychiatry 10: 975-976.

37. Dabelea D, Kinney G, Snell-Bergeon JK, Hokanson JE, Eckel RH, et al. (2003) Effect of type 1 diabetes on the gender difference in coronary artery calcification: a role for insulin resistance? The Coronary Artery Calcification in Type 1 Diabetes (CACTI) Study. Diabetes 52: 2833-2839.

38. Sacco WP, Bykowski CA (2010) Depression and hemoglobin A1c in type 1 and type 2 diabetes: the role of self-efficacy. Diabetes Res Clin Pract 90: 141-146.

39. Gendelman N, Snell-Bergeon JK, McFann K, Kinney G, Paul Wadwa R, et al. (2009) Prevalence and correlates of depression in individuals with and without type 1 diabetes. Diabetes Care 32: 575-579.

40. Shaban C, Fosbury JA, Cavan DA, Kerr D, Skinner TC (2009) The relationship between generic and diabetes specific psychological factors and glycaemic control in adults with type 1 diabetes. Diabetes Research and Clinical Practice 85: 26-29.

41. Collins MM, Corcoran P, Perry IJ (2009) Anxiety and depression symptoms in patients with diabetes. Diabet Med 26: 153-161.

42. Ahola AJ, Thorn LM, Saraheimo M, Forsblom C, Groop PH, Finndiane Study Group (2010) Depression is associated with the metabolic syndrome among patients with type 1 diabetes. Ann Med 42: 495-501.

43. Hirai FE, Tielsch JM, Klein BE, Klein R (2012) Relationship between retinopathy severity, visual impairment and depression in persons with long-term type 1 diabetes. Ophthalmic Epidemiol 19: 196-203.

44. Ahola AJ, Thorn LM, Saraheimo M, Forsblom C, Groop PH, Finndiane Study Group (2010) Depression is associated with the metabolic syndrome among patients with type 1 diabetes. Ann Med 42: 495-501.

45. Melin EO, Thunander M, Svensson R, Landin-Olsson M, Thulesius HO (2013) Depression, obesity, and smoking were independently associated with inadequate glycemic control in patients with type 1 diabetes. Eur J Endocrinol 168: 861-869.

46. Andreoulakis E, Hyphantis T, Kandylis D, lacovides A (2012) Depression in diabetes mellitus: a comprehensive review. Hippokratia 16: 205-214.

47. Pouwer F, Geelhoed-Duijvestijn PH, Tack CJ, Bazelmans E, Beekman AJ, et al. (2010) Prevalence of comorbid depression is high in out-patients with Type 1 or Type 2 diabetes mellitus. Results from three out-patient clinics in the Netherlands. Diabet Med 27: 217-224.

48. Melin EO, Thunander M, Svensson R, Landin-Olsson M, Thulesius HO (2013) Depression, obesity, and smoking were independently associated with inadequate glycemic control in patients with type 1 diabetes. Eur J Endocrinol 168: 861-869. 
Citation: Subasinghe SS, Bongetti E, O'Brien CL, Silberberg C, Ward G, et al. (2015) A Review of the Prevalence and Associations of Depression and Anxiety in Type 1 Diabetes Mellitus. J Diabetes Metab Disord 2: 007.

- Page 12 of 11 . 